\title{
La continuidad del modelo extractivista de desarrollo en el Perú
}

\author{
Jan Lust \\ Universidad Ricardo Palma, Lima, Perú \\ jan.lust @urp.edu.pe
}

\begin{abstract}
RESUMEN
El crecimiento de la exportación de los recursos naturales del país y la inversión extranjera en los sectores extractivistas son cruciales para el desarrollo de la economía peruana. Desde la década de los noventas el modelo de desarrollo económico está basado en la exportación de los commodities y la desregulación de los mercados. En este artículo argumentamos que la continuidad del modelo extractivista de desarrollo del país es la consecuencia de la interacción entre las condiciones objetivas y subjetivas del desarrollo capitalista peruano. El análisis de las condiciones objetivas se centra en el papel del país en la división internacional del trabajo, la relación entre este papel y la estructura económica y empresarial del Perú, y la funcionalidad del modelo extractivista de desarrollo para el Estado peruano. En el caso de las condiciones subjetivas, se analiza la fuerza de la izquierda política, el movimiento trabajador y los movimientos sociales contra el capital minero. La debilidad de las condiciones subjetivas hace que sea imposible cambiar las condiciones objetivas.
\end{abstract}

Palabras clave: División internacional del trabajo, estructura económica, modelo extractivista de desarrollo, resistencia

\section{The continuity of the extractivist development model in Peru}

\begin{abstract}
The growth of the exports of the country natural resources and foreign investment in the extractivist sectors are crucial for the development of Peruvian economy. Since the 1990s, the economic development model has been based on the export of commodities and the markets deregulation. In this article we argue that the continuity of the extractivist development model of the country is the consequence of the interaction between the objective and subjective conditions of the Peruvian capitalist development. The analysis of the objective conditions focuses on the role of the country in the international division of labor, the relation between this role and the economic and corporate structure of Peru, and the functionality of the extractivist development model for the Peruvian State. In the case of the subjective conditions, the force of political left, the workers movement and the social movements against the mining capital are analyzed. The weakness of subjective conditions makes it impossible to change the objective conditions.
\end{abstract}

KeYwords: International Division of Labor, economic structure, extractivist development model, resistance 


\section{Introducción}

El crecimiento de la exportación de los recursos naturales del país y la inversión extranjera en los sectores extractivistas son cruciales para el desarrollo de la economía peruana. Desde la década de los noventas, el modelo de desarrollo económico está basado en la exportación de los commodities y la desregulación de los mercados. Fue durante el régimen de Alberto Fujimori (1990-2000) que se implementó este modelo.

Los gobiernos de Alejandro Toledo (2001-2006) y Alan García (2006-2011), que vinieron después de Fujimori, no alteraron el modelo de desarrollo. El régimen de Ollanta Humala (2011-2016) adicionó al modelo extractivista proyectos que deberían proporcionar una infraestructura adecuada para un flujo más eficiente de los recursos naturales y la redistribución fiscal de la riqueza a las capas sociales que se vieron más afectadas por este modelo.

El gobierno de Pedro Pablo Kuczynski (2016-2018) tampoco introdujo cambios en el modelo de desarrollo. No solo desactivó el Plan Nacional de Diversificación Productiva (Ministerio de la Producción, 2014a) implementado por la administración de Humala, sino que también tomó medidas para acelerar las inversiones mineras. El gobierno actual de Martín Vizcarra no tiene ningún interés de cambiar el modelo.

El modelo de desarrollo peruano ha condenado el progreso económico del país a la mano visible de las crisis económicas, las fluctuaciones y los flujos del capital especulativo. Las crisis en el Norte Global tienen efectos directos sobre el Perú en el sentido de que disminuyen las inversiones del capital transnacional en los sectores extractivos (principalmente el sector minero), reducen la demanda y los precios de las materias primas del país, y ralentiza el crecimiento económico. ${ }^{1}$ Dancourt (2016, $\mathrm{s} / \mathrm{p}$ ) demuestra que existe una relación entre el crecimiento económico y los precios de los commodities. Una reducción de estos precios contribuye a generar recesiones. Gonzales de Olarte (1986, p. 15) sostiene que en el periodo 1948-1985 las crisis de corto plazo fueron causados por la reducción del valor de exportación. La recuperación económica fue consecuencia de la mejora de los precios internacionales de cobre, zinc y plata, entre otros. Por lo tanto, a la luz de la crítica conocida sobre los modelos extractivistas de desarrollo (Prebisch, 1949; Lewis, 1955; Rostow, 1961; Furtado, 1980), la continuidad de su versión peruana llama la atención. Se implementaron y siguen implementando políticas que aumentan la dependencia del país de sus sectores extractivos, especialmente del sector minero. ¿Cómo se puede explicar esto?

1 El Norte Global está conformado por los países que forman parte de la Organización para la Cooperación y el Desarrollo Económico (OCDE) y China. 
Creemos que la continuidad del modelo no puede explicarse por factores aislados. Es decir, su continuidad no es solo el resultado del poder del capital transnacional o del papel hegemónico del capital extractivo en el aparato estatal o la debilidad de las fuerzas que pretenden alterar el modelo de desarrollo vigente. Pensamos, más bien, que su continuidad es el resultado de la interacción de una variedad de factores y actores sociales, de las cuales los mencionados arriba son de importancia crucial.

Argumentaremos que la continuidad del modelo extractivista de desarrollo es la consecuencia de la interacción entre las condiciones objetivas y subjetivas del desarrollo capitalista en el Perú. Las condiciones objetivas se refieren al rol del país en la división internacional del trabajo, la relación entre este rol y las estructuras económicas y empresariales del país, y la funcionalidad del modelo extractivista de desarrollo para el Estado. Las condiciones subjetivas se relacionan con la fuerza de la izquierda política, el movimiento obrero y los movimientos sociales contra el capital minero.

Este artículo está estructurado en cinco secciones. En la primera sección examinamos la relación entre la división internacional del trabajo y las estructuras económicas y empresariales del país. La siguiente sección describe la importancia del sector minero para el Tesoro Peruano y los gastos sociales gubernamentales. En la tercera sección discutimos las debilidades de las fuerzas que intentan cambiar el modelo de desarrollo y en la sección que sigue describimos las principales políticas mineras de los gobiernos peruanos desde los años noventa. La última sección presenta nuestras conclusiones.

\section{La estructura de la economía peruana}

El modelo de desarrollo económico basado en la exportación de commodities y la inversión extranjera en los sectores extractivistas está enraizado en el rol del Perú en la división internacional del trabajo. En los años comprendidos entre 1980 y 2018, la contribución de los productos mineros al total de las exportaciones fluctuó entre 40 y $62 \% .^{2}$ El modelo extractivista de desarrollo es, por una parte, la expresión política de la función del país en el mundo capitalista globalizado.

Desde la implementación de este modelo en la década de 1990, el país no ha abandonado la agenda de inversión del capital minero transnacional. En el período 1990-1997, la inversión en exploración y explotación minera creció en 2000\% (World Bank, 2005, p. 20). En 2010, de todos los países latinoamericanos, el Perú recibió la mayor inversión en exploración minera y fue el tercero en el mundo, des-

2 Fuente: https://estadisticas.bcrp.gob.pe/estadisticas/series/anuales/resultados/PM05410BA/html (consultado $12 / 02 / 2020)$. 
pués de Canadá y Australia (Panfichi y Coronel, 2011, p. 395). En 2014, el sector minero tenía una cartera de inversiones de US\$ 61.5 mil millones, la segunda más grande de América Latina, detrás de Chile. ${ }^{3}$

Las estructuras económicas y empresariales de un país podrían considerarse, en parte, como expresiones internas de la función de un país en el mundo capitalista globalizado. Estas estructuras, sin embargo, no desempeñan un papel pasivo, ya que ayudan a fortalecer el rol de una nación en la división internacional del trabajo.

El papel del Perú como proveedor de materias primas se expresa en la reducida importancia del sector manufacturero para el Producto Bruto Interno (PBI) (en 2018, aproximadamente 12,8\%) y el hecho de que la mayoría de los bienes transables como electricidad electricidad y agua, construcción, comercio y la mayoría de los servicios, pertenecen a los sectores extractivos de la economía. La dominancia de los sectores no transables en el PBI, en 2018 aproximadamente el 68\%, es un indicador que la producción no se centra en bienes y servicios exportables de alto valor agregado. ${ }^{4}$

Los flujos internacionales de capital fortalecen la relación entre el rol del país en el mundo capitalista globalizado y la estructura económica del país. Estos movimientos no solo son el resultado de este rol de la nación en la división internacional del trabajo, sino también esta división misma ayuda a moldear, a su vez, las particularidades de estos flujos. Los movimientos de inversión extranjera directa (IED) revelan que el capital internacional ha estado, principalmente, interesado en los sectores de minería, de hidrocarburos y de telecomunicaciones. Las privatizaciones en la década de 1990 muestran que los activos más atractivos fueron justamente las empresas públicas en los mencionados sectores (Ruiz Caro, 2002, pp. 28-29).

La relación entre el papel del país en la división internacional del trabajo y los flujos internacionales de capital está expresada en la parte superior de la estructura empresarial del Perú. La revista de negocios America Economía muestra que entre los años 1992 y 2013 las principales corporaciones (según ventas) fueron aquellas que operaron en los sectores extractivos de la economía. Desde 2014, sin embargo, el capital extractivo ya no lidera el ranking de las cinco compañías más grandes que operan en el Perú. Esto es principalmente el resultado de la reducción del valor de exportación de los recursos minerales, consecuencia de la reducción de la demanda en el exterior, principalmente de China (disminución crecimiento económico). Según los datos de 2018, los sectores de hidrocarburos lideraron el ranking de las empresas

3 Fuente: «Minería peruana tiene la segunda mayor cartera de inversiones en América Latina», en http://www. americaeconomia.com/negocios-industrias/mineria-peruana-tiene-la-segunda-mayor-cartera-de-inversiones-enamerica-latina (consultado 03/10/2017); "Gold's Top 20 - Mines, miners and countries», en http://www.mineweb.com/news/gold/golds-top-20-mines-miners-and-countries/ (consultado 24/09/2015).

4 Fuente: https://estadisticas.bcrp.gob.pe/estadisticas/series/anuales/producto-bruto-interno-por-sectores-productivos-mill-soles-del-2007 (consultado 12/02/2020). 
más grandes del país. En 1992, la mayoría de estas corporaciones eran peruanas. A partir del año 2000, el capital extranjero domina las cinco principales empresas más grandes del Perú.

La mayoría absoluta de las empresas peruanas se encuentran dentro de la categoría de pequeñas y microempresas. ${ }^{5}$ Estas empresas son de crucial importancia para la población, ya que son los proveedores primordiales de empleo. Los datos del Censo Nacional de 1993 indican que el 58,7\% de la Poblacoón Económicamente Activa (PEA) ocupada (seis años o más) trabajaba en empresas (definidas como "centro de trabajo») que emplearon a menos de cinco personas y el 65,2\% en empresas de una a diez personas (INEI, 1994, p. 1561). Tres años más tarde, el 59,7\% de la PEA ocupada en zonas urbanas (de 14 años o más) trabajaba en empresas con menos de cinco trabajadores o empleados (INEI, 1997a, p. 237). En 1997, el 72\% de la PEA ocupada trabajaba en microempresas definidas como empresas que emplean menos de 10 personas (INEI, 1997b). En 2018, la situación no ha cambiado. Según la encuesta de hogares de 2018, alrededor de 78\% de la PEA ocupada trabajaba en empresas de 1 a 9 trabajadores y/o empleados.

Aunque las microempresas son cruciales para el empleo, su contribución a la producción nacional total es pequeña. En 2008, Herrera García (2011, p. 82) concluye que la contribución de las microempresas al valor agregado fue 13,9\%. Según la información del Ministerio de Producción (2017, p. 21), en 2015 la contribución de las microempresas formales ${ }^{6}$ al valor agregado del sector privado fue $6,6 \%$.

El estancamiento económico de las empresas peruanas puede demostrarse plenamente cuando comparamos los datos de 1991 y 2007 sobre el número de empresas (privadas) con los datos del período 2012-2017. En la Tabla 1 se presentan datos sobre el número absoluto de empresas privadas (registradas) según las ventas anuales para el período 2012-2017.

La mayoría de las empresas exportadoras son microempresas. Sin embargo, al igual que en el caso de su contribución a las ventas anuales, la participación de estas corporaciones al valor total de las exportaciones es insignificante. En la Tabla 2 presentamos datos referidos a las exportaciones totales según el tamaño de la empresa entre 2011 y 2017.

5 La definición de microempresas, pequeńas empresas, medianas empresas y grandes empresas se basa en las ventas anuales. Una microempresa está valuada a una tasa máxima de 150 Unidad Impositiva Tributarias (UIT). Las ventas anuales de una pequeńa empresa se encuentran entre 150 y 1.700 UIT. Las ventas de medianas y grandes empresas superan las 1700 UIT. El valor de 1 (una) UIT varía año por año. En 2020, una UIT fue igual a US\$ 1,254 .

$6 \quad$ La definición de microempresas está basada en ventas anuales. 
TABLA 1

Número absoluta de empresas privadas (registradas) según ventas: 2012-2017

\begin{tabular}{|l|l|l|l|}
\hline Año & Microempresas & Pequeñas empresas & Empresas medianas y grandes \\
\hline 2012 & $1,557,700$ & 61,322 & 9,582 \\
\hline 2013 & $1,689,366$ & 71,442 & 11,195 \\
\hline 2014 & $1,787,857$ & 77,503 & 11,380 \\
\hline 2015 & $1,933,525$ & 89,993 & 12,494 \\
\hline 2016 & $2,011,153$ & 92,789 & 13,031 \\
\hline 2017 & $2,0183,121$ & 98,942 & 13,898 \\
\hline
\end{tabular}

Fuentes: INEI (2014; 2015; 2016; 2017; 2018).

Elaboración propia.

\section{TABLA 2}

Número de empresas exportadoras y el valor de las exportaciones totales según el tamaño de la empresa: 2011-2017

\begin{tabular}{|l|c|c|c|c|c|c|c|c|}
\hline & \multicolumn{2}{|c|}{2011} & \multicolumn{2}{c|}{2012} & \multicolumn{2}{c|}{2013} & \multicolumn{2}{c|}{2014} \\
\hline & $\begin{array}{c}\text { Número } \\
\text { de } \\
\text { empresas }\end{array}$ & $\begin{array}{c}\text { Valor en } \\
\text { millones } \\
\text { de US\$ }\end{array}$ & $\begin{array}{c}\text { Número } \\
\text { de } \\
\text { empresas }\end{array}$ & $\begin{array}{c}\text { Valor en } \\
\text { millones } \\
\text { de US\$ }\end{array}$ & $\begin{array}{c}\text { Número } \\
\text { de } \\
\text { empresas }\end{array}$ & $\begin{array}{c}\text { Valor en } \\
\text { millones } \\
\text { de US\$ }\end{array}$ & $\begin{array}{c}\text { Número } \\
\text { de } \\
\text { empresas }\end{array}$ & $\begin{array}{c}\text { Valor en } \\
\text { millones } \\
\text { de US\$ }\end{array}$ \\
\hline Microempresas & 2,838 & 144 & 2,805 & 173 & 2,763 & 239 & 2,826 & 181 \\
\hline $\begin{array}{l}\text { Pequeñas } \\
\text { empresas }\end{array}$ & 2,599 & 1,075 & 2,806 & 1,246 & 2,800 & 1,294 & 2,851 & 1,203 \\
\hline $\begin{array}{l}\text { Empresas } \\
\text { medianas }\end{array}$ & 268 & 270 & 288 & 418 & 282 & 296 & 292 & 338 \\
\hline $\begin{array}{l}\text { Empresas } \\
\text { grandes }\end{array}$ & 1,864 & 40,664 & 1,893 & 40,180 & 1,932 & 36,655 & 1,960 & 33,650 \\
\hline Total & 7,569 & 42,153 & 7,792 & 42,017 & 7,777 & 38,483 & 7,929 & 35,371 \\
\hline
\end{tabular}

\begin{tabular}{|l|r|r|r|r|r|r|}
\hline & \multicolumn{2}{|c|}{2015} & \multicolumn{2}{c|}{2016} & \multicolumn{2}{c|}{2017} \\
\hline & $\begin{array}{c}\text { Número de } \\
\text { empresas }\end{array}$ & $\begin{array}{c}\text { Valor en } \\
\text { millones } \\
\text { de US\$ } \$\end{array}$ & $\begin{array}{c}\text { Número } \\
\text { de } \\
\text { empresas }\end{array}$ & $\begin{array}{c}\text { Valor en } \\
\text { millones } \\
\text { de US\$ }\end{array}$ & $\begin{array}{c}\text { Número } \\
\text { de } \\
\text { empresas }\end{array}$ & $\begin{array}{c}\text { Valor en } \\
\text { millones de } \\
\text { US\$ }\end{array}$ \\
\hline Microempresas & 2,636 & 156 & 2,536 & 151 & 3,395 & $1,651^{8}$ \\
\hline Pequeñas empresas & 2,365 & 906 & 2,375 & 899 & 1,292 & 355 \\
\hline Empresas medianas & 291 & 313 & 294 & 267 & 208 & 332 \\
\hline Empresas grandes & 2,058 & 29,612 & 2,073 & 32,990 & 2,044 & 39,086 \\
\hline Total & 7,350 & 30,986 & 7,278 & 34,307 & 6,936 & 41,424 \\
\hline
\end{tabular}

Fuentes: Ministerio de la Producción (2012; 2014b; 2017a; 2017b; 2018).

Elaboración propia.

7 La definición de micro, pequeñas y medianas y grandes empresas está basada en las ventas anuales de estas empresas.

8 Se debe observar que en 2017 el valor de las exportaciones de las microempresas ha aumentado enormemente. Nos parece extraño cuando lo comparamos con los datos de 2011 hasta 2016. También se puede visualizar la reducción significante de las exportaciones de las empresas pequeñas. No hay manera de comprobar la veracidad de todos estos datos. 
La prominencia de las micro pequeñas empresas en la economía peruana explica el tamaño reducido del mercado interno del país. El desarrollo de la economía mundial es la condición principal para su desarrollo. Es decir, el crecimiento económico como el estancamiento y el decrecimiento determina el desarrollo del mercado interno. El aumento del PBI y, en consecuencia, el crecimiento del mercado interno desde 2004, es, principalmente, el resultado de la demanda de los productos mineros del país y la IED en los sectores extractivos.

\section{El sector minero, la tesorería y los gastos sociales}

La importancia del sector minero para la economía peruana también puede demostrarse por su importancia para el Tesoro peruano. En la Tabla 3 presentamos la contribución del sector minero a los ingresos totales del impuesto a la renta en el período desde el inicio del auge de las materias primas, es decir, en 2005, hasta 2018. Es interesante observar que desde el final del auge de los commodities la contribución del sector minero al total del ingreso por impuesto a la renta ha caído considerablemente. En los últimos años parece que la tendencia hacia abajo se está revirtiéndose.

TABLA 3

Contribución del sector minero al impuesto a la renta:

2005-2018 (en porcentajes)

\begin{tabular}{|c|c|}
\hline Ańo & $\begin{array}{c}\text { Porcentaje de la contribución minera al } \\
\text { impuesto a la renta }\end{array}$ \\
\hline 2005 & $30.0 \%$ \\
\hline 2006 & $44.0 \%$ \\
\hline 2007 & $50.9 \%$ \\
\hline 2008 & $39.3 \%$ \\
\hline 2009 & $23.4 \%$ \\
\hline 2010 & $32.1 \%$ \\
\hline 2011 & $33.3 \%$ \\
\hline 2012 & $25.7 \%$ \\
\hline 2013 & $14.7 \%$ \\
\hline 2014 & $10.1 \%$ \\
\hline 2015 & $6.1 \%$ \\
\hline 2016 & $4.5 \%$ \\
\hline 2017 & $13.6 \%$ \\
\hline 2018 & $20.2 \%$ \\
\hline
\end{tabular}

Fuente: Cooperacción (2020).

Elaboración propia. 
El sector minero no solo contribuye al Tesoro Peruano a través de sus pagos del impuesto a la renta, sino también paga el Impuesto General a las Ventas y otros impuestos, como el impuesto temporal de los activos netos y el impuesto especial a la minería. Sin embargo, como indican los datos, estas contribuciones forman una parte relativamente pequeña del pago total de impuestos del sector minero. Por ejemplo, en 2002 el 74,2\% del total de los pagos de impuestos por parte del sector minero fueron impuestos a la renta. En 2011 y 2013, estos porcentajes fueron de $84.4 \%$ y 68.8\% respectivamente (Mendoza y De Echave, 2016, p. 26). Además, el impuesto general a las ventas no se aplica a los productos mineros cuando se exportan.

El crecimiento económico estimulado por el desarrollo del sector minero ha sido una de las principales fuentes para que disminuyan las tasas de pobreza (Parodi Trece, 2014, p. 324). Además, su contribución al Tesoro peruano ha sido clave para el financiamiento de los programas sociales de los diferentes gobiernos. En el Gráfico 1 demostramos la evolución de las tasas de pobreza en el periodo 2003-2017. En el Gráfico 2 se muestra la evolución de los gastos sociales en los años 2003 a 2017. La correlación entre las tasas de pobreza y los gastos sociales es clara.

\section{Gráfico 1}

Tasas de pobreza: 2004-2017 (en porcentajes)

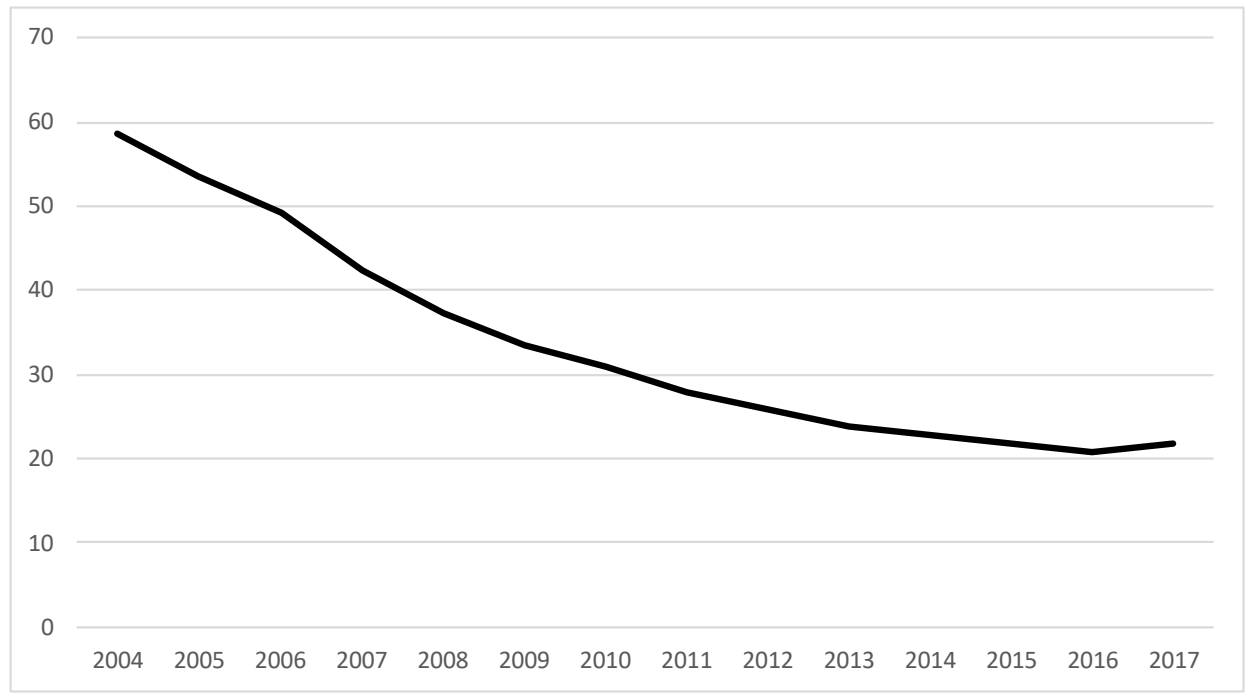

Fuente: https://www.inei.gob.pe/estadisticas/indice-tematico/sociales/ (consultado 12/09/2017 y 12/02/2020). Elaboración propia 


\section{Gráfico 2}

Gastos sociales de los programas sociales priorizados: 2003-2017 (en millones de nuevos soles)**

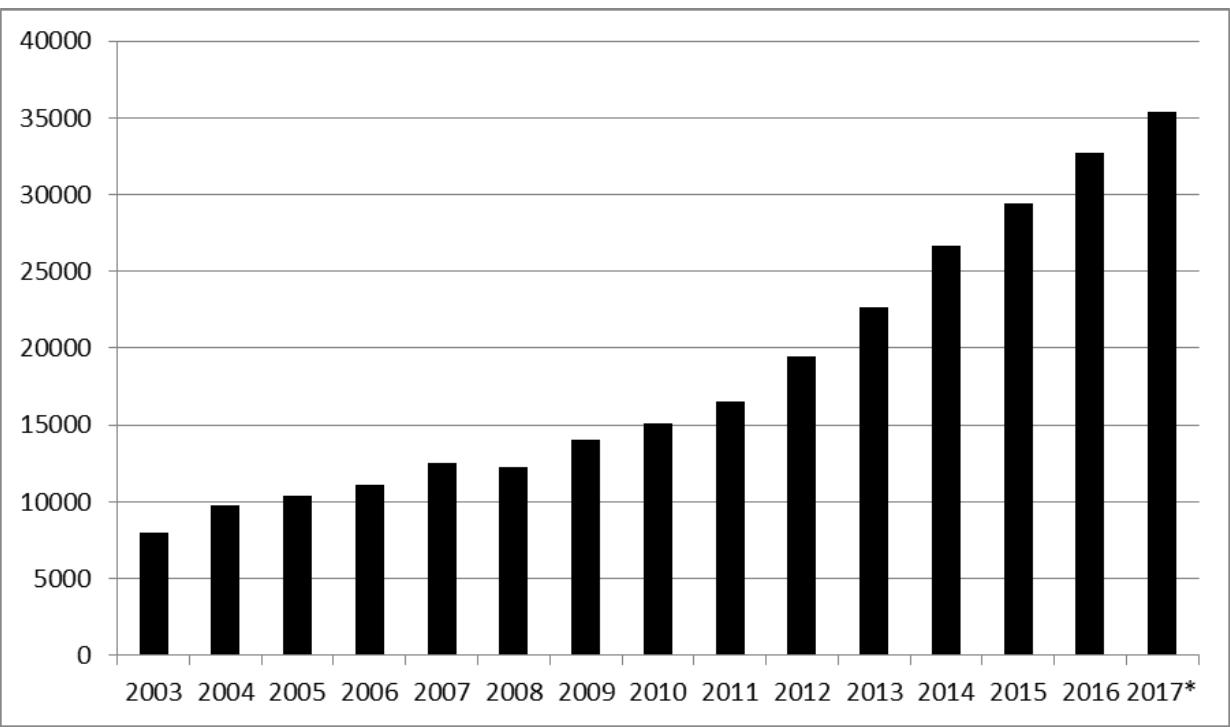

* Datos preeliminarios.

** Estos programas son educación preescolar, educación primaria, educación secundaria, promoción y atención social y comunitaria, salud colectiva y salud individual.

Fuentes: http://www.inei.gob.pe/estadisticas/indice-tematico/sociales/ (consultado 18/04/2016, 31/08/2017 y 09/05/2019).

Elaboración propia.

\section{La izquierda política peruana, los sindicatos y los movimientos sociales contra el capital minera}

Las elecciones presidenciales peruanas de 2016 no fueron sorprendentemente ganadas por un candidato (Pedro Pablo Kuczynski) que favoreció la continuación del modelo de desarrollo neoliberal implementado en los años noventa. La gran sorpresa de estas elecciones fue el regreso de la izquierda en el Congreso peruano. ${ }^{9}$ La alianza socialdemócrata Frente Amplio (FA) obtuvo el 18,7\% de los votos válidos. En las elecciones parlementarias interinas de 2020, la izquierda vi su votación reducir a alrededor de 11\% (el conjunto de Juntos por el Perú y el Frente Amplio por Justicia, Vida y Libertad).

La presencia organizada de la izquierda en el Congreso es definitivamente importante para la lucha por el cambio del modelo extractivista de desarrollo. Si bien el

9 La última presencia organizativa de la izquierda en el Congreso fue en el período 1995-2000 cuando el frente electoral de orientación socialista Izquierda Unida (IU) tenía dos escańos. 
modelo es, en gran parte, responsable del notable crecimiento del $\mathrm{PBI},{ }^{10}$ la reducción de la pobreza y el aumento del gasto público, todavía (datos de 2018) alrededor del 45\% de la PEA está subempleada, la gran mayoría de la fuerza laboral trabaja en micro y pequeñas empresas y recibe una remuneración por debajo o justo por encima del sueldo minimo, ${ }^{11}$ y la mayoría absoluta de la población activa está empleada de manera temporal. ${ }^{12}$ En otras palabras, el progreso económico no se ha filtrado realmente a todas las capas sociales.

Estos problemas, junto con las cuestiones sociales y ambientales relacionadas al sector minero, entre otros, explicaron el descontento dentro de la población que se expresa en los logros electorales de la izquierda en las elecciones presidenciales de 2016. El retroceso en 2020 es una clara expresión de sus debilidades políticas y sociales estructurales.

Los resultados de las elecciones de 2016 enmascararon que la izquierda carecía de una base política y social sólida en las ciudades. Los de 2020 demostraron que también en la parte rural del país la izquierda no tiene una presencia «estructuralmente» ganado. Todo esto se debe, principalmente, a la incapacidad de la izquierda peruana de responder estratégica y organizativamente a los cambios que han estado ocurriendo en la estructura de clases del país desde mediados de los años ochenta (Lust, 2018). Mientras que en los ańos 70 y 80 los barrios marginales y los distritos populares «pertenecían» a la izquierda, a partir de los años 90 corresponden a la derecha popular.

La debilidad política y organizativa de la izquierda puede ilustrarse por la pérdida de poder de los sindicatos cuando comparamos los años ochenta y noventa con el periodo 2000-2018. Los datos sobre la afiliación sindical muestran que mientras en 1987 el 34\% de los trabajadores del sector privado estaba afiliado a un sindicato, en 1995 la afiliación sindical había caído al 13\% (Thomas, 1999, p. 279). En la Tabla 4 presentamos datos sobre el movimiento huelgista en el period 1980-2018.

\section{TABLA 4}

Huelgas en el sector privado: 1980-2018

\begin{tabular}{|l|l|l|l|}
\hline & $1980-1990$ & $1991-1999$ & $2000-2014$ \\
\hline Huelgas & 7,612 & 1,227 & 1,315 \\
\hline Trabajadores involucrados & $5,346,638$ & 552,831 & 543,932 \\
\hline Horas hombre perdidas & $201,755,074$ & $19,120,157$ & $27,600,985$ \\
\hline
\end{tabular}

Fuente: Ministerio de Trabajo y Promoción del Empleo (2019).

Elaboración propia.

10 Según el FMI (2013, p. 5), durante el período 2000-2012, la economía casi se duplicó y el PBI real creció a una tasa promedio anual de $6,3 \%$.

11 Fuente: Instituto Nacional de Estadística e Informática, Encuesta Nacional de Hogares, 1998-2016 and http:// series.inei.gob.pe:8080/sirtod-series/ and www.mintra.gob.pe (consultado 06/08/2017).

12 Sobre los contratos temporales, ver «Los contratos temporales aumentaron antes de la sentencia del TC», en https://elcomercio.pe/economia/contratos-temporales-aumentaron-sentencia-tc-noticia-514003 (consultado el 24/08 / 2018). 
La disminución del poder sindical es, en general, la consecuencia de los cambios en la distribución del empleo. Estos cambios fueron, primordialmente, el resultado de las crisis en la década de 1980. Los trabajadores anteriormente asalariados, comenzaron a establecer pequeños negocios y/o empezaron a trabajar por cuenta propia, principalmente en el sector informal. Mientras en 1993 el 65,2\% de la PEA ocupada (seis ańos o más) ) trabajó en empresas que empleaban de una a 10 personas (INEI, 1994, p. 1561); en 1999, el 72,3\% del PEA ocupada en zonas urbanas (14 años y más) trabajaba en estas empresas (INEI, 2010, p. 81). En 2018, el 72,4\% de la PEA era informal. ${ }^{13}$

La concentración de la población trabajadora en pequeńas empresas hace que sea muy difícil organizarlas. Los sindicatos no están permitidos en empresas que emplean a menos de 20 personas y la negociación colectiva solo está permitida en algunas ramas del sector público, la construcción y la minería. Los trabajadores independientes no están organizados en sindicatos.

En la década de noventa, el poder sindical se vio muy afectado por la privatización de las empresas estatales y los despidos masivos en el sector público. También el uso generalizado de los contratos temporales tuvo un impacto negativo sobre los sindicatos. Las personas con un contrato temporal no se afilian a un sindicato (Yepez del Castillo y Bernedo Alvarado, 1985, p. 84; Fernández, entrevista, 2015). Los sindicatos parecen no ser de utilidad para estos trabajadores, ya que sus contratos son de corta duración. Los datos de Thomas (1999, p. 276) muestran que mientras en 1985 el 38\% de la fuerza laboral trabajaba en base a contratos temporales, en 1990 esto aumentó a 41\% y en 1995 ya había crecido a 50\%. En 2003, el 77\% de la población económicamente activa fue contratada de forma temporal. En 2012, la mayoría de los contratos en cualquier tipo de empresa y de cualquier tamańo fueron contratos temporales (Instituto de Estudios Sindicales, 2012, p. 3).

En los últimos diecinueve años, los movimientos sociales anti-mineros no han podido cambiar el modelo de desarrollo debido a tres debilidades fundamentales. La primera debilidad es el carácter local de la lucha y su proyección local (Auris, entrevista, 2015). Según De Echave (2009, p. 16), los movimientos sociales indígenas y campesinos organizados localmente contra el capital minero podrían ser efectivos para responder a los conflictos, problemas y casos locales, pero no pueden articular una agenda nacional referida a la minería.

La segunda debilidad tiene que ver con la falta de vínculos entre los movimientos contra el capital minero en las áreas rurales y las organizaciones sociales en las áreas urbanas. Aunque estas relaciones podrían existir, no hay evidencia de una alianza estratégica entre los movimientos y las organizaciones en ambas áreas.

13 Fuente: https:/www.inei.gob.pe/estadisticas/indice-tematico/ocupacion-y-vivienda/ (consultado 12/02/2020). 
La tercera debilidad es el hecho de que los movimientos sociales contra el capital minero no parecen tener una estrategia que pueda permitir que estos movimientos determinen el curso de la batalla. Los movimientos solo reaccionan a lo que sucede en su entorno directo en lugar de tratar de determinar el curso de los eventos futuros. Las actividades de las corporaciones mineras y las medidas tomadas por el gobierno que favorecen el capital minero determinan las acciones de los movimientos sociales.

\section{Políticas gubernamentales y el capital minero}

Desde la década de 1990, los diferentes gobiernos han implementado políticas que cumplían plenamente con el rol del Perú en el mundo capitalista globalizado. El Perú no debería tener la intención de volverse en contra de su ventaja comparativa en el comercio internacional, sino que, más bien, debe aprovecharse de esta ventaja.

Durante el gobierno de Fujimori, el sector minero se vio beneficiado a través de la implementación de leyes que le permitieron apropiarse de las tierras de las comunidades. La Ley General de Minería de 1992 hizo posible y legal la reasignación de comunidades y poblaciones con fines mineros (Domínguez, 2010, p. 24). La liberalización del mercado de tierras en 1995 proporcionó, entre otras, las condiciones legales para la división de las tierras de las comunidades indígenas y campesinas en parcelas individuales.

Las medidas económicas y desregulatorias tomadas por el régimen de Fujimori incluían pactos de estabilidad jurídica con las empresas, y mecanismos legales especiales, como la depreciación acelerada, la posibilidad de deducir inversiones en infraestructura pública de pagos de impuestos, la exención de impuestos hasta que la inversión inicial se había recuperado o si los ingresos generados se habrían utilizado para hacer reinversiones con el fin de aumentar la producción en más del 10\% y la deducción de los costos de investigación y exploración minera de los pagos de impuestos (Campodónico Sánchez, 1999, pp. 17 -24). Durante los próximos 10 a 15 años se prohibió cambiar las leyes que protegían los intereses del capital. Las concesiones mineras se otorgaron por un período indefinido y el capital solo tenía que pagar US\$ 2 al año (por hectárea) para mantener sus derechos de concesión (Campodónico Sánchez, 1999, p 57en10).

El gobierno de Toledo promovió el modelo extractivista de desarrollo en el sentido de que el régimen promulgó un Decreto Ley Supremo que reducía los derechos de importación pagados sobre aquellos bienes de capital que se iban a utilizar en la exploración y la producción de ciertos minerales como el petróleo y el gas en la región amazónica, y una ley que eliminó el impuesto a las ventas del 18\% sobre bienes de capital y servicios para la exploración de minerales (Gurmendi, 2010, p. 17.3). 
Además de estimular los sectores de la minería y de los hidrocarburos, debe mencionarse que a partir de la administración de Toledo las compañías mineras estaban obligadas a pagar regalías por el uso de los recursos minerales del país. Estas regalías fueron completamente transferidas a los gobiernos regionales y locales. Las corporaciones que habían firmado pactos de estabilidad jurídica no necesitaban pagar estas regalías. Las regalías se consideran costos y pueden deducirse de los pagos del impuesto a la renta. Como el $50 \%$ de los pagos del impuesto a la renta de las empresas mineras se transfiere a los gobiernos regionales y locales, el efecto neto de la posibilidad de deducir regalías implica una reducción de los pagos totales de transferencia a estos gobiernos y una relativa disminución de los ingresos fiscales del gobierno central.

Las políticas implementadas por el gobierno de García pueden reanudarse en sus esfuerzos para acelerar el proceso de parcelación de las tierras de campesinos y de indígenas, y para proporcionar títulos de propiedad individual de estas tierras. El régimen intentó romper las comunidades haciendo uso de los poderes legislativos que se otorgaron para implementar el acuerdo de libre comercio con los Estados Unidos. ${ }^{14}$ Además del «programa de regalías», el gobierno implementó el Programa de Solidaridad Minera con el Pueblo. Este programa consistió en una contribución voluntaria de las compañías mineras a un fondo privado, en gran parte administrado por las propias corporaciones, que tenía como objetivo financiar proyectos para mejorar las condiciones de vida de la población en las áreas mineras. Las compañías que aceptaron participar en este programa tuvieron que donar el $2 \%$ de sus ganancias, después del impuesto a la renta y antes del pago de dividendos. ${ }^{15}$

La administración de Humala trató de encontrar respuestas a las consecuencias económicas del fin del auge de los commodities. Se implementaron políticas que deberían atraer nuevas inversiones del capital minero (nuevos pactos de estabilidad legal para proteger a las compañías mineras por cambios en el régimen tributario) y aumentar la producción minera (medidas que deberían acelerar el proceso de aprobación de concesiones mineras, reducción de las regulaciones ambientales). Para aumentar sus capacidades para financiar sus programas sociales, se modificó el programa de regalías que se introdujo en 2004. Aunque cambió la metodología en base a la cual se calculó cuánto debían pagar las compañías (a las compañías se les permitió deducir más costos antes de pagar la regalía), también introdujo dos nuevos «regímenes de impuestos mineros» (ambos deducibles de la impuesta a la renta). Además, el

14 La resistencia de la población indígena en la región amazónica ayudó a revocar estas leyes, pero terminó en una masacre. La represión de las protestas en la ciudad de Bagua (2010) causó la muerte de 23 policías y 10 civiles.

15 Según Mendoza y De Echave (2016: 41), eran solo ocho empresas que contribuían el 80,7\% a este fondo privado. Todas ellas no pagaban regalías mineras. 
gobierno logró aumentar el número de corporaciones que pagaban impuestos por el uso de los recursos minerales del país.

La principal contribución del gobierno Kuczynski a la continuidad del modelo de desarrollo basado en la extracción de recursos naturales fue, como decimos en la introducción de este artículo, la desactivación del Plan Nacional de Diversificación Productiva. La administración de Vizcarra no ha introducido ningún cambio de carácter estructural en el modelo extractivista de desarrollo.

\section{Conclusiones}

La continuidad del modelo extractivista de desarrollo es producto de una variedad de factores interrelacionados y actores sociales. Si bien este modelo se basa en el papel Perú en el mundo capitalista globalizado, la continuidad del modelo no puede atribuirse únicamente a la función del país en la división internacional de trabajo. Además, la necesidad de accumular capital y la urgente necesidad de China de los minerales del país tampoco pueden explicarnos toda la historia detrás de la continuidad del modelo. Sin embargo, atribuimos a estos factores un papel determinante en la combinación de factores que «deciden» sobre la continuidad o discontinuidad del modelo de desarrollo implementado.

En este artículo argumentamos que la continuidad del modelo extractivista de desarrollo es el resultado de la interacción entre las condiciones objetivas y subjetivas del desarrollo capitalista en el país. En términos concretos, nos referimos a la función del Perú en la división internacional del trabajo, las estructuras económicas y empresariales del Perú, la funcionalidad del modelo para el Estado y la fuerza de la izquierda política, el movimiento laboral y los movimientos sociales contra el capital minero.

El papel del país como proveedor de materias primas es la razón principal por la cual la estructura económica del país está dominada por sectores no transables. Los países en el Norte Global y, especialmente, el capital transnacional, no están particularmente interesados en el desarrollo de otros sectores y ramas. La falta de este interés se demuestra en los flujos de capital que se dirigen principalmente hacia aquellos sectores que refuerzan el rol del Perú en la división internacional del trabajo. Por lo tanto, no es sorprendente que la parte superior de la estructura empresarial peruana esté ocupada por corporaciones que operan en los sectores extractivos de la economía.

Las microempresas son fundamentales para el empleo, pero no contribuyen significativamente al PBI y su participación en el valor de las exportaciones totales es insignificante. Si bien el papel del Perú en la división internacional del trabajo es, en 
gran parte, responsable por la presencia dominante de estas empresas en la sociedad, también el modelo extractivista de desarrollo ha contribuido a esta situación al reforzar la función del país en el capitalismo globalizado.

La importancia del sector minero para el crecimiento económico y su importante contribución al Tesoro (y gastos sociales) fueron en los últimos quince años los argumentos clave para que los responsables de la política peruana fortifiquen el actual modelo de desarrollo y, como tal, fortalezcan el papel del país en la división internacional del trabajo. Sin embargo, debe subrayarse que la implementación de políticas gubernamentales en beneficio del capital extractivo no es tanto la consecuencia automática de esta función, sino la expresión de una correlación de fuerzas de clase dentro de la sociedad que favorece los intereses del capital extractivo.

Las condiciones objetivas a favor del modelo extractivista de desarrollo no hacen, en sí mismas, imposibles de cambiar el modelo. La continuidad del modelo extractvivista de desarrollo se explica por la debilidad de las condiciones subjetivas para alterar las condiciones objetivas. Esta debilidad es, en parte, el resultado del desarrollo particular de las condiciones objetivas. La izquierda política y los sindicatos carecen de las bases políticas y sociales para convertir las fuerzas de clase en los protagonistas y defensores de un modelo de desarrollo sostenible. Esta debilidad política y organizativa, unida a las debilidades de los movimientos sociales contra el capital minero, ayuda a mantener el equilibrio político a favor del modelo extractivista de desarrollo.

\section{Referencias}

Campodónico Sánchez, H. (1999). Las reformas estructurales en el sector minero peruano y las características de la inversión 1992-2008. Chile, ECLAC, Serie Reformas Económicas 24. Recuperado de: http://repositorio.cepal.org/bitstream/handle/11362/7471/1/ S9900588_es.pdf.

Cooperacción (2020). Actualidad Minera del Perú n. 247. Recuperado de: http://cooperaccion. org.pe/wp-content/uploads/2020/01/Bolet\%C3\%ADn-AMP-ENERO-2020.pdf.

Dancourt, Ó. (2016), Las vacas flacas en la economía peruana. Documento de Trabajo 428. Lima: PUCP, Departamento de Economía.

De Echave, J. (2009). Los retos actuales del movimiento social vinculado a la lucha por los derechos de las comunidades frente a las industrias extractivas: el caso peruano. Recuperado de: http:// www.yorku.ca/cerlac/EI/papers/De\%20Echave.pdf.

Dominguez, C. (2010). The water is MINE! Negotiation and resistance between Andean communities and the MINE (Tesis Magíster, Law Governance Group). Wageningen University, Wageningen, The Netherlands. 
FitzGerald, E.V.K. (1981). La economía politica del Perú, 1956-1978. Desarrollo económico y reestructuración del capital. Lima: Instituto de Estudios Peruanos.

Furtado, C. (1980). La economía latinoamericana. Formación histórica y problemas contemporáneos. Mexico: Siglo Veintiuno Editores S.A.

Gonzales de Olarte, E. (1986). Crisis y democracia. El Perú en busca de un nuevo paradigma de desarrollo. Documento de Trabajo 21, Serie Economía. 6. Lima: Instituto de Estudios Peruanos. Recuperado de http://lanic.utexas.edu/project/laoap/iep/ddt021.pdf.

Grupo Propuesta Ciudadana. (2014). Vigilancia de las industrias extractivas. Reporte Nacional no. 18, Perú 2013. Lima, Grupo Propuesta Ciudadana. Recuerpado de: http:/www.propuestaciudadana.org.pe/sites/default/files/publicaciones/archivos/Reporte\%20VIE\%2018. pdf.

Gurmendi Alfredo C. (2012). The mineral industry of Peru. En U.S. Department of the Interior and U.S. Geological Survey, 2010 Minerals Yearbook (pp. 17.1-17.14). Recuperado de http:// minerals.usgs.gov/minerals/pubs/country/2010/myb3-2010-pe.pdf.

Herrera García, B. (2011). Análisis estructural de las MYPEs y PYMES. Quipukamayo. Revista de la Facultad de Ciencias Contables, (18: 35), pp. 69-89. Doi: https://doi.org/10.15381/ quipu.v18i35.3706.

INEI. (2018). Perú: Estructura empresarial 2017. Lima: INEI.

INEI. (2017). Perú: Estructura empresarial 2016. Lima: INEI.

INEI. (2016). Perú: Estructura empresarial 2015. Lima: INEI.

INEI. (2015). Perú: Estructura empresarial 2014. Lima: INEI.

INEI. (2014). Perú: Estructura empresarial 2013. Lima: INEI.

INEI. (2010). Perú: Evolución de los indicadores de empleo e ingresos por departamentos, 20012009. Lima: INEI.

INEI. (2008). IV Censo Nacional Económico 2008. Perú: Características económicas de las micro y pequeñas empresas en el año 2007. Lima: INEI.

INEI. (1997a). Perú: Comportamiento del empleo urbano 1995-96. Encuesta Nacional de Hogares. Lima: INEI.

INEI (1997b), «Perfil del trabajador de la pequeña y micro empresa 1997». Recuperado de http://proyectos.inei.gob.pe/web/biblioineipub/bancopub/Est/Lib0164/INDICE.htm.

INEI. (1994). Censos Nacionales 1993. IX de Población. IV de Vivienda. Resultados Definitivos. Perú, Tomo II. Lima: INEI.

INEI. (1993). Perú: Compendio estadistico 1992-1993. Tomo I. Lima: INEI.

Instituto de Estudios Sindicales. (2012). La situación laboral y sindical en el Perú. Serie: Documentos de Trabajo 1. Recuperado de: https://www.iesiperu.org.pe/documentos/ LASITUACIONLABORALYSINDICALENELPERU.pdf

International Monetary Fund. (2013). IMF Country Report. n . 13/45. Recuperado de: http:// www.imf.org/external/pubs/ft/scr/2013/cr1345.pdf. 
Lewis, A. W. (1955). Teoría del desarrollo económico. Mexico / Bogota: Fondo de Cultura Económica.

Lust, J. (2018). Capitalism, class and revolution in Peru, 1980-2016. Chaim: Palgrave MacMillan. Mendoza, A. y De Echave, J. (2016). ¿Pagaron lo justo? Política fiscal peruana en tiempos del boom minero. Lima: CooperAcción / Oxfam.

Ministerio de Trabajo y Promoción del Empleo. (2019). Anuario Estadístico Sectorial 2018. Lima: Ministerio de Trabajo y Promoción del Empleo.

Ministerio de la Producción (2018), Las MYPYME en cifras 2017. Lima: Ministerio de Producción.

Ministerio de la Producción. (2017a). Las MYPYME en cifras 2015. Lima: Ministerio de Producción.

Ministerio de la Producción. (2017b). Las MYPYME en cifras 2016. Lima: Ministerio de Producción.

Ministerio de la Producción. (2014a). Plan nacional de diversificación productiva. Documento sujeto a consulta pública. Lima, Ministerio de Producción. Recuperado de: https://www.mesadeconcertacion.org.pe/sites/default/files/archivos/2015/documentos/11/mp_plan_nacional_de_diversificacion_productiva_2014.pdf.

Ministerio de la Producción. (2014b). Las MYPYME en cifras 2013. Lima: Ministerio de Producción.

Panfichi, A. y Coronel, O. (2011). Los conflictos hídricos en el Perú 2006-2010: una lectura panorámica. En R. Boelens, L. Cremers y M. Zwarteveen (coords.), Justicia Hidrica. Acumulación, conflicto y acción social (pp. 393-422). Lima, Justicia Hídrica / Instituto de Estudios Peruanos / Fondo Cultural PUCP.

Parodi Trece, C. (2014). Perú 1995-2012. Cambios y continuidades. Lima: Universidad del Pacífico.

Prebisch, R. (1950). "The Economic Development of Latin America and its principal problems»,. Recuperado de http://www.rrojasdatabank.info/prebisch_theec-development.pdf.

Rostow, W.W. (1961). Las etapas del crecimiento económico. Un manifiesto no comunista. Mexico / Buenos Aires: Fondo de Cultura Económica.

Ruiz Caro, A. (2002). El proceso de privatizaciones en el Perú durante el periodo 1991-2002, Santiago de Chile, Instituto Latinoamericano y del Caribe de Planificación Económica y Social (ILPES), Serie de Gestión Pública, no. 22. Recuperado de: http://repositorio.cepal. org/bitstream/handle/11362/7273/S027489_es.pdf?sequence=1.

Thomas, J. (1999). El mercado laboral y el empleo. En J. Crabtree y J. Thomas (coords.), El Perú de Fujimori: 1990-1998 (pp. 255-296). Lima: Universidad del Pacífico / Instituto de Estudios Peruanos.

Torres Cuzcano, V. (2013). Grupos económicos y bonanza minera en el Perú. El caso de cinco grupos mineros nacionales. Lima: CooperAccion. 
World Bank. (2011). The World Bank Group in Extractive Industries. 2011 Annual Review. Washington D.C. Recuperado de: http://siteresources.worldbank.org/INTOGMC/ Resources/WBG_EI_Annual_Report_FY11_Final.pdf.

World Bank. (2005). Wealth and sustainability: The environmental and social dimensions of the mining sector in Peru. Peru Country Management Unit, Environmentally and Socially Sustainable Development, Latin America and the Caribbean Region, Washington D.C. Recuperado de: http://siteresources.worldbank.org/INTPERUINSPANISH/Resources/ AAA_Environment_and_Mining_in_Peru.pdf.

Yepez del Castillo, I. y Bernedo Alvarado, J. (1985). La sindicalización en el Perú. Lima: Fundación Friedrich Ebert, Pontificia Universidad Católica.

\section{Entrevistas}

Auris, Olmedo, un cuadro del Partido Comunista del Perú - Patria Roja y Vicepresidente del Movimiento de Afirmación Social, Lima, 31 de marzo de 2015.

Fernández, Ibis, un líder de la Confederación General de Trabajadores del Perú, Lima, 29 de mayo de 2015. 